\title{
Persistence of amoebiasis in northern Sri Lanka - a public health failure
}

\author{
Kannathasan $\mathbf{S}^{1}$, De Silva $\mathbf{N} \mathbf{R}^{2}$, Kumanan $\mathbf{T}^{3}$ \\ Journal of the Ceylon College of Physicians, 2017, 48, 88-89
}

The Ceylon Medical Journal recently published a case report of acute fulminant necrotizing colitis, a deadly complication of amoebiasis caused by Entamoeba histolytica, from northern Sri Lanka ${ }^{1}$. Another recent paper reported 346 molecular and immunologically confirmed patients with amoebic liver abscess, all seen at a single centre (Teaching Hospital, Jaffna) within a period of 3 years ${ }^{2}$.

Amoebiasis is an ancient disease, however, still prevalent in the tropics, even in the $21^{\text {st }}$ century. Availability of newer laboratory tests for confirmation of diagnosis has provided an opportunity to study this age-old disease and its consequences to a greater depth.

The first publication relating to hepatic amoebiasis in Sri Lanka dates back to $1962^{3}$. Several other papers on hepatic amoebiasis in Sri Lanka were published in the $1970 \mathrm{~s}^{4-7}$. Together, these articles suggested that amoebiasis and amoebic liver abscess (ALA) were serious public health issues during that period. Since then, however, the occurrence of amoebiasis in the southern parts of Sri Lanka appears to have declined quite markedly, while northern Sri Lanka continued to record a high number of cases. However, the political unrest, which prevailed in the North over the last few decades, prevented the conduct of research into this important health issue.

During the last few years, research on amoebiasis in Sri Lanka has stepped up again. An epidemiological study of 110 patients with ALA from the Northern region revealed that $97 \%$ of the study population drank palmyrah toddy; over $80 \%$ obtained their drinking water from unprotected wells; $88 \%$ drank toddy at taverns; and $80 \%$ practiced open air defecation at the taverns ${ }^{8}$.

\footnotetext{
${ }^{1}$ Department of Parasitology, University of Jaffna, Sri Lanka.

${ }^{2}$ Department of Parasitology, University of Kelaniya,Sri Lanka.

${ }^{3}$ Department of Medicine, University of Jaffna,Sri Lanka.
}

Corresponding author: Kumanan T

E-mail: mtkumanan@yahoo.com
The study on ALA patients seen at Teaching Hospital, Jaffna during the period 2012-2015, also found that a vast majority were males with a history of heavy alcohol consumption ${ }^{2}$. This study also demonstrated that the incidence of ALA at the Teaching Hospital, Jaffna had a distinct seasonal pattern, with a peak during the inter-monsoonal period from March to August. This pattern was mirrored by palmyrah toddy sales in Jaffna and lends support to the suspected role of toddy consumption in unhygienic taverns in the continued transmission of $E$. histolytica in the Jaffna peninsula. It is also noteworthy that none of the ALA patients were found to be excreting $E$. histolytica cysts in their faeces nor has there been a reported increase in the number of cases of amoebic dysentery.

E. histolytica has a relatively straight forward life cycle and mode of transmission. The parasite exists in two stages: a hardy, multinucleate, cyst form and the motile, trophozoite stage. Humans are the natural hosts of the parasite. Infection is acquired by ingestion of cysts contained in faecally contaminated food or water or, more rarely, through oral-anal sexual contact ${ }^{9}$. Cysts survive stomach acidity and excyst within the small intestine to form the 20-50 $\mu \mathrm{m}$ trophozoite stage. E. histolytica trophozoites may live in the large-bowel lumen without causing disease, or may invade the intestinal mucosa, causing amebic colitis and dysentery. In some individuals, trophozoites invade through the mucosa to enter the blood stream, travel through the portal circulation, and parasitize the liver to cause amoebic liver abscesses. Motile trophozoites excreted in the stools form an important aspect in the diagnosis. However, they are rapidly killed upon exposure to air or stomach acid and therefore are not responsible for transmission ${ }^{9}$. Trophozoites encyst within the large bowel, and the cysts which are excreted in the stool, continue the life cycle. Hence, symptomatic or asymptomatic carriers play a pivotal role in transmission.

Preventive strategies should aim at identifying the human reservoir of infection in order to interrupt the cycle of transmission. Considering the number of patients admitted to Teaching Hospital Jaffna with ALA, the number of individuals who are infected with E. histolytica, including asymptomatic carriers, could be very high. Unhygienic practices at the toddy taverns 
are likely to play a key role. Almost all the ALA patients admitted open air defecation at toddy taverns, which have no latrine facilities. The utensils used to drink and store toddy are stored in a manner that exposes them to insects like ants, flies, cockroaches etc. Water to wash these utensils, as well as clean drinking water was not available in many taverns. Improving sanitation at these toddy taverns by preventing open air defecation, screening for infection and treating carriers and upgrading the environment by providing adequate clean water resources would definitely have an impact on reducing transmission of infection. The relevant public health authorities must take appropriate urgent, practical and effective steps to control this devastating condition in the region.

\section{References}

1. Raviraj S, Sivashankar M, Ekanayake A. A rare and potentially fatal complication of amoebiasis: acute fulminant necrotizing amoebic colitis. Ceylon Med J. 62(1): 63-4.

2. Kannathasan S, Murugananthan A, Kumanan T, et al. Amoebic liver abscess in northern Sri Lanka: first report of immunological and molecular confirmation of aetiology. Parasit Vectors 2017; 10(1): 14. doi: 10.1186/s13071-016-1950-2.

3. Rajasuriya K, Nagaratnam N. Hepatic amoebiasis in Ceylon. J Trop Med Hyg. 1962; 65: 165-78.

4. Ramachandran S, Sivalingham S, Perumal JR. Hepatic amoebiasis in Ceylon. J Trop Med Hyg. 1972; 75(2): 23-33.

5. Canagaretna C. Amoebic liver abscess - a clinical study. Ceylon Med J. 1974; 19(1): 18-27.

6. Ramachandran S. Radiological changes in left lobe amoebic liver abscesses. Postgrad Med J. 1974; 50(589): 689-93.

7. Ramachandran S. The Marcus Fernando Memorial Oration 1974. Hepatic amoebiasis. Ceylon Med J. 1975; 20(2): 69-81.

8. Janani T, Pushpana P, Surenthirakumaran R, et al. Amoebic liver abscess: An emerging threat in northern Sri Lanka. EMBO global lecture course and symposium on amoebiasis: Exploring the biology and pathogenesis of Entamoeba. March 4-7, 2012. Khauraho, India pp 80.

9. Samuel L, Stanley, Jr. Amebiasis and infection with free living amoebas in Harrison's Principles of Internal Medicine, 18 ${ }^{\text {th }}$ ed, Longo, Fauci et al. 2012. 